\title{
Fatigue in Mechanochemical Systems
}

\author{
Yoichi TATARA \\ Department of Engineering, Shizuoka University, 3-5-1 Johoku, \\ Hamamatsu, Japan.
}

(Received May 19, 1977)

\begin{abstract}
Fatigue of swelling-contraction cycles of ionic networks was experimentally demonstrated for cross-linked poly(methacrylic acid) and a set of particles (ion-exchange resins). The mechanisms of fatigue in both mechanochemical systems were investigated.

KEY WORDS Fatigue / Swelling-Contraction Cycles / Ionic Networks /

Mechanochemical Systems/Poly(methacrylic acid)/Ion-Exchange

Resins /
\end{abstract}

Polyelectrolyte gels are sometimes referred to as mechanochemical systems. Like muscle and collagen, which are biological mechanochemical systems, they can convert chemical energy directly into mechanical energy. When immersed in sodium hydroxide solution, a synthetic polymer swells owing to interaction among polymeric ions and counterions, and contracts upon neutralization with hydrochloric acid. It also shows a change in size and shape according to the concentration of salt in the solution in which it is immersed. Muscle and collagen also have this property. ${ }^{1}$

The mechanochemical systems have another advantage, namely, that they can act as substances for artificial muscles. The values of force and work exerted by a unit self-weight of the polymers are similar in the same order as those of muscle or collagen., ${ }^{2,3}$

Synthetic mechanochemical systems can be obtained with filaments of poly(acrylic acid) or poly(methacrylic acid) cross-linked with poly(vinyl alcohol). Since W. Kuhn ${ }^{4}$ and A. Katchal$\mathrm{sky}^{5}$ originally synthesized these polymers, the mechanochemical systems have been studied from the standpoint of ionic interaction, ${ }^{6-10}$ phase equilibrium, ${ }^{11}$ or rubber elasticity. ${ }^{12,13}$ There have been industrial treatments such as mechanical elements using collagen, ${ }^{14}$ or ion-exchange resins. ${ }^{15}$ But, few studies have been done on the dynamics ${ }^{16}$ or the fatigue of these systems. The study of fatigue of the mechanochemical systems will be important in terms of both material science and engineering.

\section{FATIGUE OF FILAMENT}

A filament of poly(methacrylic acid) cross-linked with poly(vinyl alcohol) in the weight ratio $1: 5$ was used as the sample for the fatigue test of contraction-swelling cycles. This preparation was similar to previous papers. ${ }^{4,12,13}$ The synthesized specimen was $4 \mathrm{~cm}$ in length, $1.4 \mathrm{~cm}$ in width, and $0.011 \mathrm{~cm}$ in thickness, when swollen in pure water. It was suspended vertically between two plastic clamps in a glass vessel, with the lower clamp fixed. A thread fastened to the upper clamps was passed over two pulleys and a potentiometer, and was attached to a load of net weight $10 \mathrm{~g}$. The loaded length of the sample between the clamps was $c a$. $2 \mathrm{~cm}$ in $\mathrm{HCl}$ solution. Changes in length were recorded through the potentiometer to a recorder.

The sample demonstrated continuous cycles of contraction and swelling through periodic exchanges of $\mathrm{HCl}$ and $\mathrm{NaOH}$ solutions with each concentration of $0.1 \mathrm{~mol} / l$. The stroke for lifting the load was $c a .0 .5 \mathrm{~cm}$. It took about $30 \mathrm{sec}$ for the sample to attain each equilibrium. During the experiment, 1 to $5 \mathrm{~min}$ were taken as the intervals for solution exchange.

After about 100 cycles, we found that, sometimes, slightly inferior responses occurred; that is, both an increase in the time required to attain 
equilibrium and a decrease in the stroke were observed. At night, the sample was left in $\mathrm{NaOH}$ solution and suspended to the load (let us call this state "pause"). On the following day, the first several responses were found to recover to the level of those on the preceding day. But, by repeating the cycles, we recognized the reappearance of inferior responses. Due to the second pause on the next night, the first several responses on the third day were also better than those observed at the last stage on the second day but worse than those at the first stage on the same day, and inferior responses of the stroke and the speed developed very slightly over the following three days.

After 500 cycles, the sample at times did not contract to lift the load, until mechanical shocks were given by striking the glass vessel by hand. Several successive shocks yielded stepwise contractile responses. Whereas with 500-600 cycles, irregular and less periodic cycles continued with the introduction of intermittent shocks. At 600 cycles, the sample could neither contract nor swell, inspite of the shocks received. But no apparent mechanical fractures for the sample were recognized.

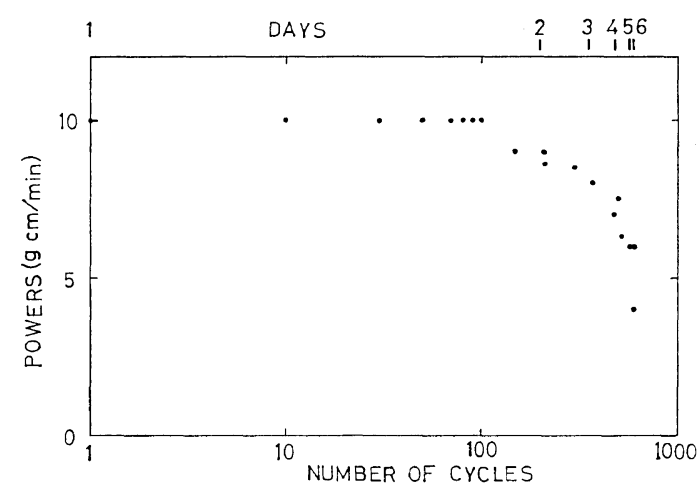

Figure 1. Changes in powers exerted by a filament of poly(methacrylic acid) cross-linked with poly(vinyl alcohol) against the numbers of the swelling-contraction cycles and against days.

This experimental result is plotted in Figure 1 as a function of powers required until the attainment of equilibrium against the progressive number of cycles; in the case of irregular responses of strokes and speeds after 100 cycles, the minimum value of the powers among 10 successive cycles was plotted in Figure 1.
This experiment shows that the present sample manifests reversible cycles of contraction and swelling within at least 100 cycles, and that pauses and mechanical shocks are effective for the temporary recovery of decreases in both the stroke and the speed and for the insensitivity to the responses, regarded here as fatigue.

\section{FATIGUE OF PARTICLES IN A CYLINDER}

A set of ion-exchange resins (Dowex 50W, X2, 50-100 mesh) was used for fatigue test of mechanochemical particles. A polycarbonate cylinder (internal diameter $30 \mathrm{~mm}$ ) having an outlet at the bottom was set vertically, and a glass filter was inserted on the bottom. The particles of a total volume of $15.3 \mathrm{~cm}^{3}$ in distilled water were packed on the lower glass filter in the cylinder. A load of $1.4 \mathrm{~kg}$ was placed on the particles through an upper glass filter.

The mechanical behavior and attempts at industrial application of this type under the name of the mechanochemical piston have already been reported. ${ }^{3,15,17}$ The cycles of swelling and contraction of the particles were repeated by periodic injections of distilled water and $\mathrm{HCl}$ solution with a concentration of $2 \mathrm{~mol} / l$ through the upper glass filter, and recorded through the potentiometer to the recorder, in a manner similar to the case of the filament-like mechanochemical system. The decompression of $-8 \mathrm{cmHg}$ at the outlet was taken for the development of passage of liquid through the particles.

The experiment was carried out during 21 days, and at night time, the particles were left in the swollen state in the cylinder (let us also call this state "pause"). For the first 300 cycles, we observed a constant response, i.e., about $4 \mathrm{~mm}$ in the stroke (its elongation ratio 1.3) and about $6 \mathrm{sec}$ in its time constant (i.e., time to attain $63-\%$ equilibrium). Thereafter, a slight decrease in stroke and speed occurred. Mechanical shocks were not effective, but pauses of leaving the particles swollen and loaded at nights were sometime effective for temporary increase in stroke or speed. When the cycles exceeded 700, speeds were remarkably decreased, as seen in Figures 2 and 3. The pauses for several hours or days at this stage were ineffective for the recovery of the responses. The cycles continued till about 850 cycles, maintaining 


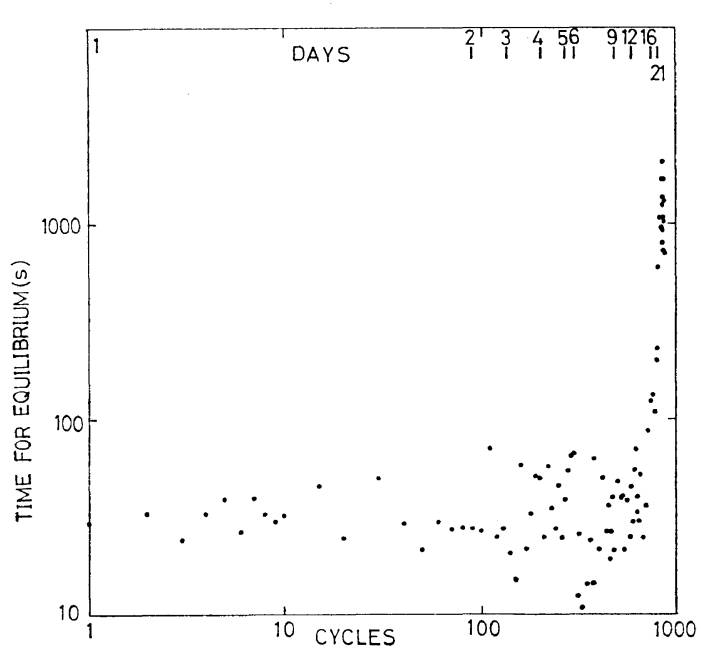

Figure 2. Changes in time for the attainment of swelling equilibrium for the cylindrical set of particles in terms of the numbers of the swelling-contraction cycles and against days.

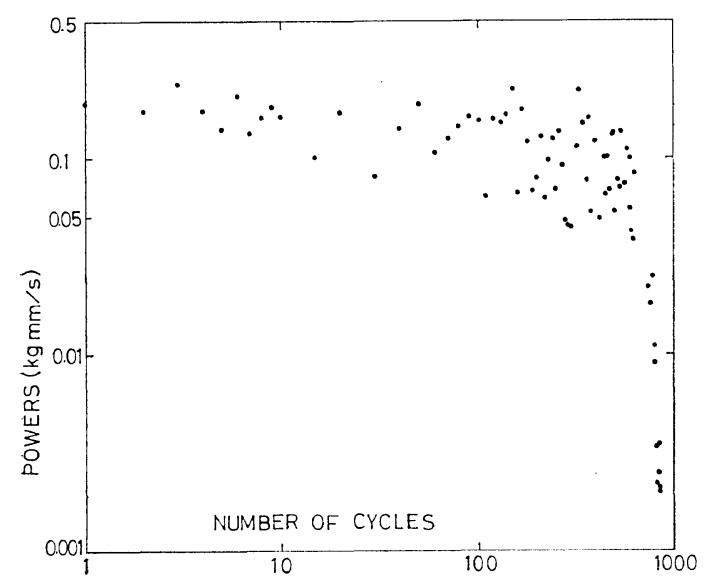

Figure 3. Changes in powers for the data of Figure 2.

ca. $50 \%$ of the stroke and $1 \%$ of the speed of the initial responses.

At the end of the experiment, some particles in the upper part in the cylinder were picked up and observed with a microscope. A photograph was shown in Figure 4. Some were found to be in a state of mechanical fracture, with others retaining their initial appearance. Thus, the remarkable decrease in speed was considered mainly due to these fractures, since the crushed particles must hinder the flow of solutions among the particles

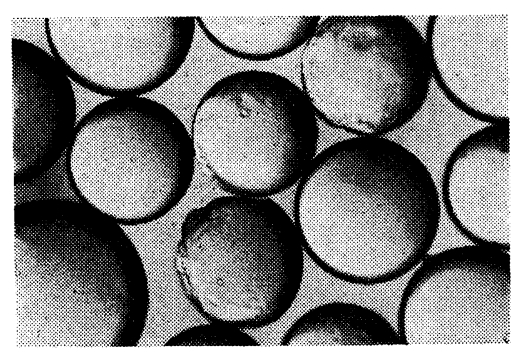

Figure 4. Examples of particles after the fatigue test of $c a .850$ cycles.

in the cylinder.

Another experiment was conducted for 20 days, using the cylinder, load of $0.34 \mathrm{~kg}$, and other fresh particles (Dowex 50W, X2, 50-100 mesh, 15.3 $\mathrm{cm}^{3}$ swollen in water). The results of fatigue test were similar to those of the present experiment. Hence, the effects of loads on fatigue are considered to be less than those of frictional restrictions against isotropic swelling of the particles packed in the rigid cylinder.

\section{CONSIDERATION ON FATIGUE MECHANISMS}

\section{Crosslinked Poly(methacrylic acid)}

According to the above experiment, the stroke and the speed of the contraction-swelling cycle were found to be simultaneously decreased with repeating cycles. The following several possible causes may be assumed for the decrease in the stroke or the speed.

I. Causes for decrease in the stroke.

A. Decrease in polymeric ions due to (1) their separation from the chains; (2) their combination with ions other than $\mathrm{H}^{+}$or $\mathrm{Na}^{+}$.

B. Decrease in the speed. (When one measures the change in length of a sample whose speed is decreased, one may possibly observe the decrease in the stroke in a given time.)

C. Scission of the chains.

II. Causes for decrease in the speed.

a. (1) Decrease in polymeric ions; (2) decrease in counterions.

b. Increase in thickness of a diffusion layer ("film") as a diffusion barrier. (It has been established $^{18}$ that such processes are diffusioncontrolled: the rate-determining mechanism is the interdiffusion of two ion species, either in the 
Nernst diffusion layer adherent to the polymer surface or within resin particles.)

c. Increase in impurities like $\mathrm{FeO}_{2}, \mathrm{Fe}_{2} \mathrm{O}_{3}$, etc. (This will lead to a decrease in the diffusion constants of $\mathrm{H}^{+}$or $\mathrm{Na}^{+}$.)

On the other hand, considering the process of the present experiment, we may exclude A (2) and C of the above causes, for A (2) since pure water was used in the preparation of the sample and during the course of the experiment, and for $\mathrm{C}$ since the fracture in the sample did not occur and the sample maintained elasticity to support the load at the last stage of the experiment.

Thus, A (1)-a (1), B-a (1), B-a (2), B-b, and $\mathrm{B}-\mathrm{C}$ may be possible causes of the fatigue, considering the simultaneous decreases in speed and stroke. Among these, a (2) may be possibly derived from cause $b$, since the diffusion layer may screen the counterions from the external solution. Cause $\mathrm{c}$ may not be cancelled out, since there is the possibility that ion $\mathrm{Fe}^{+++}$or its oxidized substance are originated from chemical combination between $\mathrm{HCl}$ solution and such metals as the wires in the plastic clamps in the apparatus.

Hence, this leaves A (1)-a (1), B-a (1), B-b, and $B-c$. In other words, the separation of polymeric ions, the increase in thickness of the diffusion layer, and the increase in the impurities due to $\mathrm{Fe}^{++}$are considered to be the causes for fatigue.

Let us discuss these causes from other standpoints of view. The present results of mechanical shocks and the pauses serving for the temporary recovery of the fatigue will also be important for investigating the fatigue mechanism, as well as the simultaneous decreases in stroke and speed mentioned above. Mechanical shocks will have the effect of stirring solutions in which the sample is immersed. According to Helfferich, ${ }^{18-20}$ the speed of ion exchange is increased for film in which exchange is controlled by diffusion and insensitive for particle in which exchange is controlled by diffusion of stirring solutions. Moreover, according to Figures 5 and 6, both contractile and swelling speed (or reverse number of time periods required to attain equilibrium) are found to be proportional to the solution concentration below $0.1 \mathrm{~mol} / \mathrm{l}$. The speed of ion exchange is proportional to solution concentrations for film-controlled exchange and independent of these for particle-controlled exchange. ${ }^{19,20}$ The swelling and contractile speeds

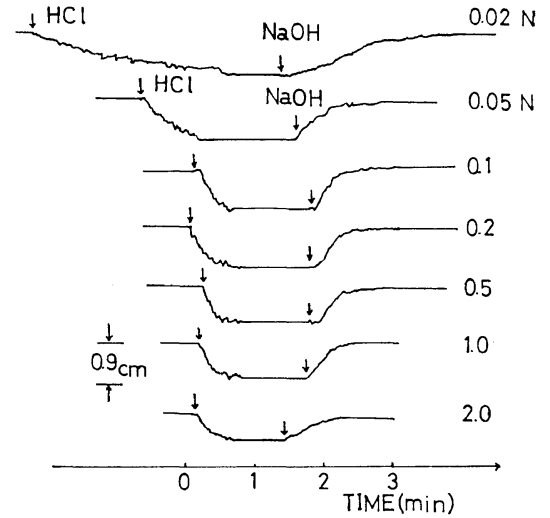

Figure 5. Contraction-swelling processes for another sample of poly(methacrylic acid) cross-linked with poly(vinyl alcohol) with the weight ratio 1: 5 for various concentrations of solutions; size of the sample, $4.5 \times 2.0 \times 0.011 \mathrm{~cm}^{3}$ swollen in water; load, $3 \mathrm{~g}$; curing condition, $5 \mathrm{hr}$ at $120^{\circ} \mathrm{C}$.

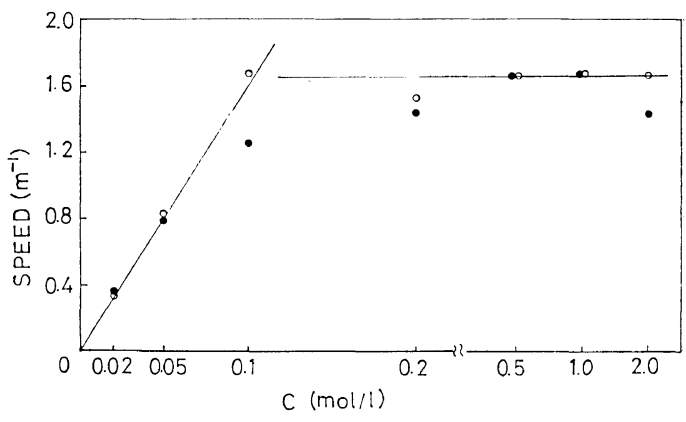

Figure 6. Speed (reverse number of time minutes required to attain equilibrium) of swelling or contraction of poly(methacrylic acid) against concentrations $\mathrm{C}$ of $\mathrm{HCl}$ or $\mathrm{NaOH}$ solution for the data of Figure 5: $\mathrm{O}$, concentration process due to $\mathrm{HCl}$; ๑, swelling process due to $\mathrm{NaOH}$.

are considered proportional to the speed of the ion exchanges controlled. ${ }^{16}$ For these two reasons, the sample with a concentration of $0.1-\mathrm{mol} / l \mathrm{HCl}$ or $\mathrm{NaOH}$ is considered to be controlled by the film-controlled exchange. Thus, the increase in thickness of the diffusion layer will cause a decrease in the speed and the stroke, and shocks will contribute to a decrease in the thickness or the breaking of part of it.

The temporary effectiveness of the pause has a rheological meaning. By leaving the polymer in the swollen state over a given time interval, the con- 
tacts among the segments or the entanglements among the chains will be partially loosened, or such frictional effects will be weakened. Moreover, the polymeric ions separated from the segments and inside the polymer should tentatively combine with neighboring segments. However, this combination may not be so strong as the original one and separation will occur again due to frictional action.

The assumption of the separation of the polymeric ions from the chains has the following basis: The network structure of cross-linked poly(methacrylic acid) or poly(acrylic acid) in sodium hydroxide or hydrochloric acid solution may be formed in the crystalline or partially crystalline state. ${ }^{13}$ Thus, accompanying the separation of the swelling and the contraction, the segments of the networks will undergo some frictional restriction by neighboring chains and by the segments nearly parallel to them. As a result, polymeric ions or ionic groups attached to the side chains will not be so difficult to separate from the segments due to the frictional effects. The separated polymeric ions will drift inside or rest on the surface of the polymer, and they will combine with or screen counterions from the external solution.

In summary, the main fatigue mechanism is considered here to be the separation of polymeric ions from the chain segments due to the frictional effect, and the auxiliary mechanism will be an increase in the thickness of the diffusion layer covering the polymer due to the drifted polymeric ions and the increase in the impurities like the oxidized ions. The latter two causes will merely contribute to decreases in the speed and the stroke of the cycle.

In the last stage of the fatigue test at which the polymer demonstrated no response, there are no ionic groups. From this viewpoint, mechanochemical systems having ionic groups embedded in the skeletal chains should be less susceptible to fatigue.

\section{Particles in the Cylinder}

There may be several different fatigue mechanisms between the above filament of poly(methacrylic acid) and the ion-exchange resins packed in the rigid cylinder, although the decreases in the stroke and the speed in the present experimental results were rather similar. One of the differences is the diffusion processes to be controlled. Figures

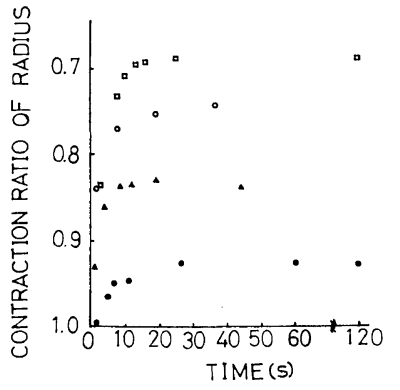

Figure 7. Contraction process of particles (Dowex $50 \mathrm{~W}, \mathrm{X} 2$ ) for various concentrations of $\mathrm{HCl}$ solution: $\square, 12-\mathrm{mol} / l \mathrm{HCl}$, diam, $5.42 \times 10^{-2} \mathrm{~cm}$; O, 9-mol $/ l \mathrm{HCl}$, diam, $5.31 \times 10^{-2} \mathrm{~cm} ; \triangle$, 4-mol $/ l$ $\mathrm{HCl}$, diam, $5.19 \times 10^{-2} \mathrm{~cm} ; \bullet, 1-\mathrm{mol} / \mathrm{l} \mathrm{HCl}$, diam, $5.40 \times 10^{-2} \mathrm{~cm}$. These data were obtained from the measurement of diameters of photographed particles, using a microscope with a camera.

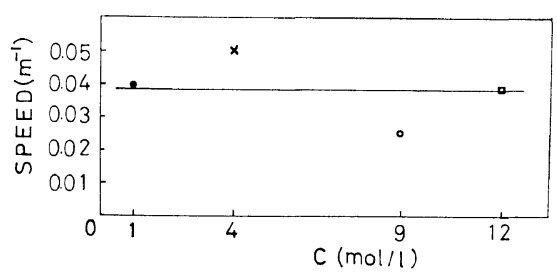

Figure 8. Speed (reverse number of time minutes required to attain equilibrium) of contraction of particles against concentrations $\mathrm{C}$ of $\mathrm{HCl}$ solution for the data of Figure 7.

7 and 8 demonstrates the experimental results of contraction against the time for ion-exchange resins (Dowex 50W, X2) using a microscope, a camera, and a projector; the contractile speed is independent of concentrations of $\mathrm{HCl}$ solution from 1 to $12 \mathrm{~mol} / l$. Thus, the contraction and swelling of ion-exchange resins with a concentration of solution of $2-\mathrm{mol} / l \mathrm{HCl}$ in the experiment are considered to be controlled by particle diffusion-controlled process. This must be due to the particle-diffusion process in which mechanical shocks were not available.

Ratio of cross-linkages is also different between the two substances; the poly(methacrylic acid) cross-linked with poly(vinyl alcohol) was $1: 5$, and the ion-exchange resin was $2 \%$. Thus, the ionexchange resin is considered not to be crystalline in structure but to have the same structure as 
ordinary elastomers. Combining this property of high elasticity with the isotropic and radial swelling and contraction of the spherical body, we may expect less microscopic frictional behavior among chains and segments in the resin than in the filament with repeating cycles. Hence, the separation of polymeric ions will occur less.

The present particle system is the cylindrical set which can stretch along the axis of the vessel when swollen. From this restriction of the spherical deformation of each particle, shearing stresses and frictional forces will act among the particles and between the particles and the vessel wall. As a result, with the swelling of the particles, mechanical fractures must have occurred, as shown in Figure 4. Fractured particles have little elasticity and obstruct the passage of solutions among the particles. These are the causes for the decrease in stroke and speed, respectively.

Temporary recovery due to the pause is considered to have arisen rather from the increase in the packing ratio of the particles due to the decrease in the swelling ratio of each particle at night when the particles in the vessel were somewhat dried. The reliefs of frictional effects between neighboring segments would be supplemental.

In summary, the main fatigue mechanism of the present particle system is the mechanical fracture due to friction among particles or between particles and the vessel wall.

A fatigue test for one particle was not treated here owing to the experimental difficulty. This main fatigue mechanism is, however, expected from the present discussions to be microscopic friction such as that between chain segments and such as separation of polymeric ions from the chains in the filament. But, as mentioned above, due to its radial swelling and contraction, it will be less susceptible to fatigue than the filament.

\section{REFERENCES}

1. L. Mandelkern, Ann. Rev. Phys. Chem., 15, 421 (1964).

2. W. Kuhn, A. Ramel, and D. H. Waters, "Size and Shape Changes of Contractile Polymers," A. Wassermann, Ed., Pergamon Press, New York, N. Y., 1960, Chapter II, p. 41.

3. Y. Tatara, Bull. Jpn. Soc. Mech. Eng., 15, 58 (1972).

4. W. Kuhn, Experientia, 5, 318 (1949).

5. A. Katchalsky, Experientia, 5, 319 (1949).

6. A. Katchalsky, O. Kunzle, and W. Kuhn, $J$. Polym. Sci., 5, 283 (1950).

7. A. Katchalsky, J. Polym. Sci., 7, 393 (1951).

8. A. Katchalsky and I. Michel, J. Polym. Sci., 15, 69 (1955).

9. S. Asakura, N. Imai, and F. Oosawa, J. Polym. Sci., 13, 499 (1954).

10. H. Morawetz, "Macromolecules in Solution," Interscience Publishers, New York, N. Y., 1969, Chapter 7.

11. P. J. Flory, "Principles of Polymer Chemistry," Cornell Univ. Press, Ithaca, N. Y., 1953, Chapter 13.

12. Y. Tatara, Bull. Jpn. Soc. Mech. Eng., 15, 49 (1972).

13. Y. Tatara, J. Polym. Sci., Polym. Symp. Ed., No. 54, 283 (1976).

14. M. V. Sussmann and A. Katchalsky, Science, 167, 45 (1970).

15. Y. Tatara, Bull. Jpn. Soc. Mech. Eng., 17, 451 (1974).

16. Y. Tatara and M. Mori, Trans. Jpn. Soc. Mech. Eng. 38, 734 (1972).

17. Y. Tatara, Kobunshi, 23, 520 (1974).

18. F. Helfferich and M. S. Plesset, J. Chem. Phys., 28, 418 (1958).

19. F. Helfferich, "Ion Exchange," McGraw Hill, New York, N. Y., 1962, p 285.

20. T. Seiyama and T. Yamabe, "Ion Exchange Resins," Nikkan Kogyo, Tokyo, 1963, p 77. 